Purdue University

Purdue e-Pubs

CTRC Research Publications

Cooling Technologies Research Center

$1-1-2004$

\title{
Experimental Investigation of the Thermal Performance of Piezoelectric Fans
}

Tolga Acikalin

Sydney M. Wait

S V. Garimella

Purdue University, sureshg@purdue.edu

Arvind Raman

Follow this and additional works at: http://docs.lib.purdue.edu/coolingpubs

Acikalin, Tolga; Wait, Sydney M.; Garimella, S V.; and Raman, Arvind, "Experimental Investigation of the Thermal Performance of Piezoelectric Fans" (2004). CTRC Research Publications. Paper 50.

http://dx.doi.org/10.1080/01457630490248223

This document has been made available through Purdue e-Pubs, a service of the Purdue University Libraries. Please contact epubs@purdue.edu for additional information. 
Submitted for Publication in Heat Transfer Engineering; Prepared with the T\&F Journal Template.

\title{
Experimental investigation of the thermal performance of piezoelectric fans
}

\author{
Tolga Açikalin \\ Sydney M. Wait \\ Suresh V. Garimella \\ Arvind Raman \\ Cooling Technologies Research Center \\ School of Mechanical Engineering \\ 585 Purdue Mall \\ Purdue University \\ West Lafayette, Indiana 47907-2088 USA
}

Address Correspondence to: Professor Suresh V. Garimella, sureshg@ecn.purdue.edu, Tel: (765) 494-5621 


\begin{abstract}
Piezoelectric fans are investigated as a cooling technology for the thermal management of electronic devices. Flow visualization experiments are conducted to better understand the physics of fan operation. Prototypes of the fans are built and tested to assess their feasibility and cooling performance, and to determine optimal locations for the fans. An enclosure the size of a cellular phone, and a commercially available laptop computer, are used for demonstrating the cooling feasibility of the fans. Piezoelectric fans are found to offer significant localized cooling, exceeding enhancements in convective heat transfer coefficients of 100\%,, while exhibiting low power consumption, minimal noise, and small dimensions. Performance metrics for piezoelectric fans should be based on heat transfer characteristics, such as the percent increase in the heat transfer coefficient of the system. Optimization techniques that maximize the electromechanical coupling factor (EMCF) can be used to design efficient fans.
\end{abstract}

\title{
Introduction
}

Advances in portable electronics to meet increasing consumer demand for smaller products with greater functionality have resulted in faster and more powerful electronic components to be installed into smaller spaces in such devices. Although conventional methods of heat removal, such as incorporation of heat sinks and rotational fans, are thermally viable, it is the minimized space, power, and noise requirements that point to piezoelectric fans as an alternate method of thermal management. Piezoelectric fans are not designed to replace traditional rotational fans and heat sinks in larger devices, but can be used to supplement cooling in stagnant areas and hot spots where the cooling of the rotational fan is less effective.

In this study, piezoelectric fans are investigated as an active cooling technique for the thermal management of portable electronics. The two most remarkable characteristics of the piezoelectric fans are their low acoustic noise levels and their low power consumption. These features make piezoelectric fans well-suited for applications in the thermal management of portable electronic devices.

A piezoelectric fan is fabricated by bonding a piezoelectric patch or several patches to a shim material cut to the desired shape and size. If only one patch is used, this patch may be oriented in any direction on either side of the fan. However, in a two-patch configuration (one on each side) the patches are oriented such that when one expands, the other contracts. When an alternating voltage is applied to the piezoelectric patch, it expands and 
contracts alternately with the same frequency as the input signal. As this happens, the blade (shim material) bonded to the piezoelectric patch flaps back and forth like a Japanese fan (Figure 1), albeit much faster.

The alternating voltage is applied at the frequency of the $\mathrm{r}^{\text {st }}$ resonance mode of the piezoelectric fan. Driving the fan at resonance minimizes the power consumption of the fan while providing maximum tip deflection. To ensure silent operation, the fans are designed such that their first mode of resonance will be at the lower limit or outside the range of frequencies audible to the human ear $(<100 \mathrm{~Hz})$. Although it is possible to run the piezoelectric fans at higher modes of resonance, this is not preferred in view of the fact that the frequencies corresponding to these mode shapes fall in the audible range for relatively small-sized fans $(1-5 \mathrm{~cm})$.

\section{Previous Work}

Toda [1,2] experimented with a piezoelectric fan that was fabricated from eight, layered sheets of $\mathrm{PVF}_{2}$, each $9 \mu \mathrm{m}$ thick. This multi-layered bimorph fan was $3.5 \mathrm{~cm}$ long, $9.5 \mathrm{~cm}$ wide and was clamped at one end in a cantilever fashion. When driven by an input signal with an amplitude of $140 \mathrm{~V}$ and a frequency of $13 \mathrm{~Hz}$, the fan produced a $1.4 \mathrm{~m} / \mathrm{s}$ airflow velocity at the tip, and bulk airflow of 4.5 liters/s. In a thermal experiment, two of these fans on either side of a power transistor panel of a television receiver resulted in a $17^{\circ} \mathrm{C}$ decrease in temperature on the surface of the panel. The temperature of the inner space of the receiver was also reduced by $5^{\circ} \mathrm{C}$.

Yoo et al. [3] developed several types of piezoelectric fans, one of which was fabricated out of phosphor bronze with a PZT patch length of $3.3 \mathrm{~cm}$, total length of $6.5 \mathrm{~cm}$, width of $2.6 \mathrm{~cm}$ and thickness of $0.01 \mathrm{~cm}$. An input signal with amplitude $110 \mathrm{~V}$ and frequency $60 \mathrm{~Hz}$ resulted in a fan tip deflection of $3.55 \mathrm{~cm}$ and an air velocity of $3.1 \mathrm{~m} / \mathrm{s}$ measured $0.1 \mathrm{~cm}$ away from the fan tip.

Schmidt [4] performed experiments to determine the local and average transfer coefficients on a vertical surface cooled by two piezoelectric $\left(\mathrm{PVF}_{2}\right)$ fans resonating out of phase. The piezoelectric fans used in the experiments were $1.75 \mathrm{~cm}$ wide, $4.13 \mathrm{~cm}$ long and were separated by a distance of $3.38 \mathrm{~cm}$. Mass transfer rates, in terms of the nondimensional local Sherwood numbers, were obtained from measurements of surface contours of naphthalene-coated plates both before and after exposure to the fans. The maximum local Sherwood number was achieved when the fans were placed $0.318 \mathrm{~cm}$ from the vertical surface. Schmidt found that varying the distance between the fans and the surface, and the distance from one fan to another, noticeably changed the transfer coefficients for the system. 
Loh et al. [5] investigated the cooling effects of acoustic streaming from an ultrasonically vibrating beam $12.8 \mathrm{~cm}$ long, $1.0 \mathrm{~cm}$ wide and $0.1 \mathrm{~cm}$ thick. A heat source dissipating $3.4 \mathrm{~W}$ was placed $0.15 \mathrm{~cm}$ above the nonvibrating beam and a steady-state temperature of $98^{\circ} \mathrm{C}$ was reached in the heater with the ambient temperature being $20^{\circ} \mathrm{C}$. The beam was then vibrated at a resonant frequency of $28 \mathrm{kHz}$, with a vibration amplitude of $10 \mu \mathrm{m}$. The steady-state temperature for the heat source decreased from $98^{\circ} \mathrm{C}$ to $68^{\circ} \mathrm{C}$. By increasing the vibration amplitude to $25 \mu \mathrm{m}$, the heater temperature dropped further to $58^{\circ} \mathrm{C}$. Nyborg's theory [6] was also used in this study to generate predictions for the streaming flow, against which CFD simulations of this beam were compared.

Buermann et al. [7] performed an optimization study of a piezoelectric fan with two symmetrically placed piezoelectric patches. An analytical Bernoulli-Euler model and a finite element model of the composite piezo-beam were used in analyzing the piezoelectric fan. A closed-form analytical solution was developed for the piezoelectric fan, and optimal patch-to-blade length and patch-to-blade thickness ratios were calculated for maximizing the electromechanical coupling factor (EMCF), tip deflection and rotation, and stroke volume rate.

Ihara and Watanabe [8] investigated flows around the ends of oscillating flexible cantilevers. In order to simplify the problem, the oscillating plates were sandwiched between parallel plates, rendering the flow twodimensional. The discrete vortex method, in conjunction with the singularity method was used to numerically simulate the flow field. Flow visualization experiments were conducted to validate the numerical simulation.

\section{Current Work}

Feasibility studies of piezoelectric fans in small-scale electronics cooling applications were performed. Flow visualization experiments were conducted to gain insight into the flow induced by these fans. The thermal performance of piezoelectric fans was investigated in two different experiments: a custom-built setup was used to quantify the cooling enhancement from a heat-dissipating component due to piezoelectric fans in an enclosure simulating a cellular phone, and a commercially available laptop computer was utilized to demonstrate the viability of using piezoelectric fans for localized cooling. The noise level of the fans was not measurable because the background noise in the lab environment, approximately $30 \mathrm{~dB}$, was greater than that produced by the fans.

The fans fabricated for the present work were constructed using $0.13 \mathrm{~mm}$ thick brass or $0.076 \mathrm{~mm}$ thick steel for the shim stock and $0.19 \mathrm{~mm}$ thick PZT for the piezoelectric patch. Since the PZT is a highly capacitive material, the power consumption of the piezoelectric fans is very low $(<10 \mathrm{~mW})$. Two prototype piezoelectric fans are shown in Figure 2. 


\section{Flow Visualization}

The flow generated by the piezoelectric fans can be very complex and needs to be well-understood for the fans to be incorporated into cooling systems. In flow visualization experiments conducted towards this end, water droplets from a humidifier were used to seed the flow, and were illuminated with an Argon-ion laser sheet.

A $5 \mathrm{~cm}$ long piezoelectric fan made from a stainless steel shim was placed in a laptop-sized box of dimensions $29 \mathrm{~cm} \times 24 \mathrm{~cm} \times 4 \mathrm{~cm}$, built from clear acrylic for the purpose of visualization. The resonance frequency of this fan was approximately $20 \mathrm{~Hz}$ for the $1^{\text {st }}$ mode; at this frequency and an input of $40 \mathrm{~V}$, a $1.5 \mathrm{~cm}$ tip deflection is achieved. The fan was placed such that the tip of the fan was close to the right wall of the box as shown in Figure 3. A sheet of light was introduced from the right side, parallel to the top and bottom surfaces of the box, and the camera was placed directly above, for viewing through the top surface. Figure 4 shows the transient flow generated by the vibrating fan. At $\mathrm{t}=1 / 30 \mathrm{~s}$ (Figure $4 \mathrm{a}$ ) the fan has already made a downward sweep and is heading back up towards the upper extreme position. At this instant a vortex (A) is clearly seen to be shed. At $t=$ $2 / 30 \mathrm{~s}$ (Figure $4 \mathrm{~b}$ ) the fan is at its lower extreme position for the second time. The boundary layers around the fan are seen to start forming a distinct pattern. Another vortex (B) is shed, this time on the upper side of the fan, while the first one (A) is displaced downwards.

At $\mathrm{t}=3 / 30 \mathrm{~s}$ (Figure $4 \mathrm{c}$ ) the fan is passing through the neutral position. At this instant, it is apparent that vortex $B$ is moving clockwise while vortex $A$ is moving counter-clockwise. At $t=5 / 30 \mathrm{~s}$, Figure $4 \mathrm{~d}$ shows the displacements of vortices A and B, with A being further along than B. The boundary layer formed over the fan is clearly visible and the flow patterns seen at this instant suggest that the vortices are shed slightly before the tip of the fan.

Two additional vortices are observed $(C$ and $D)$ at $t=8 / 30 \mathrm{~s}$ (Figure $4 \mathrm{e}$ ). In Figures $4 \mathrm{f}$ and $4 \mathrm{~g}$, the vortices continue to emerge and move away from the point of origin. At $t=15 / 30 \mathrm{~s}$, the boundary layers over the fan appear to be fully developed and unchanging with time (Figure 4h). As time passes, vortices of opposite circulation continue to be shed from each side of the fan, traveling in opposite directions.

Streamlines deduced from the visualizations at a time that the flow has reached a developed state are shown in Figure 5. Suction occurs both near the clamp on the left and near the tip on the right. The time-averaged flow field is symmetric about the fan. Two circulation zones having opposite directions are observed on each side of the fan (counter-clockwise on top, clockwise on the bottom). The maximum fluid rejection velocity is observed slightly 
before the tip of the piezoelectric fan. This velocity, estimated from the flow visualizations, was approximately 30 $\mathrm{cm} / \mathrm{s}$.

\section{Heat Transfer Experiment: Cell Phone Cooling}

In order to assess the performance of the piezoelectric fans, an enclosure with dimensions similar to those of a cell phone, $11.5 \mathrm{~cm} \times 5.7 \mathrm{~cm} \times 3.8 \mathrm{~cm}$, was used. The fan used in this experiment had dimensions of $6.35 \mathrm{~cm} \times$ $1 \mathrm{~cm}$ and was made of a stainless steel shim. These parameters resulted in a resonant frequency of $20 \mathrm{~Hz}$ and vibration amplitude of $1.5 \mathrm{~cm}$ under a $40 \mathrm{~V}$ input. The enclosure was open on either end to provide an inlet and outlet for the air flow, as shown in Figure 6, though the inlet of the enclosure was partially blocked by the clamp of the piezoelectric fan. All other surfaces were insulated using polystyrene foam to simulate adiabatic boundary conditions. The piezoelectric fan was tested first in a vertical orientation (stroke being from side to side), and then in a horizontal orientation (stroke being up and down). The fan oriented in the vertical configuration was tested in three different positions near the heat sink, and the fan in the horizontal orientation was tested in one position to one side of the heat sink. Figure 6 shows the details of these configurations.

The heat source used in the experiment consisted of a special kapton heater embedded in a copper block, as described in [9]. A heat sink was attached to the heat source to allow for a heat dissipation rate of $2 \mathrm{~W}$, maintained the same in all the experiments, without overheating the heat source.

When the temperatures in the domain showed no variation over a period of 1 hour it was assumed that steady-state operating conditions had been reached. The convective heat transfer coefficient in each case (natural convection and with the fans placed in different positions) was calculated by using an energy balance between the power input and the heat removed from the surface of the heat source by convection according to:

$$
h=\frac{q}{A\left(T_{\text {chip }}-T_{\text {avg }}\right)}
$$

In equation (1), $q$ is the power input to the heat source, $A$ is the exposed surface area of the copper block (it may be noted that the heat transfer coefficient is always referenced to the surface area of the heat source, and not that of the heat sink, in this work), and $T_{a v g}$ is the average temperature from three thermocouples used to measure the air temperatures over the heat sink at different locations. Another thermocouple was used to monitor the temperature of the copper block heat source $\left(T_{\text {chip }}\right)$. Measurements were first obtained under natural convection conditions, with 
the fan remaining off. Then, with the piezoelectric fan turned on in different positions, the system was allowed to reach new steady-state temperatures for the heat source and surrounding air at the same input power, and the new heat transfer coefficient was calculated. The heat transfer coefficients for the four different piezoelectric fan positions studied are plotted in Figure 7. The uncertainties in measured heat transfer coefficients were estimated to be under $8 \%$ for all the experiments.

It is apparent that the largest heat transfer enhancement was obtained when the fan covered half of the heat sink, i.e., in position 1 . In this position there was more than a $100 \%$ increase in the heat transfer coefficient due to the fans, relative to natural convection alone. The corresponding temperature differences between the heat source and the surroundings were $24.1^{\circ} \mathrm{C}$ under natural convection and $11.9^{\circ} \mathrm{C}$ with the fan turned on. For fan position 3 , the enhancement in heat transfer coefficient was limited to $52 \%$. When the fan was vibrating in the horizontal orientation instead of the vertical, the enhancement in heat transfer was $28 \%$ (position 4). For the best case, the heat transfer enhancement of greater than $100 \%$ implies that the heat transfer rate, in a setup such as the one under study, can be doubled (for instance, from $1 \mathrm{~W}$ in natural convection alone to $2 \mathrm{~W}$ with the fan) by a fan that has a power consumption of approximately $3 \mathrm{~mW}$.

\section{Heat Transfer Experiment: Laptop Cooling}

Yet another promising application area for piezoelectric fans is in the thermal management of laptop computers, where fan noise levels and power consumption are two important criteria. A feasibility study was conducted to evaluate piezoelectric fans as a thermal management solution for a commercially available laptop computer. Two different configurations were used in this experiment. In the first (Setup 1), a wide fan and a narrow transverse fan were both placed near the power electronics in the laptop, and in the second (Setup 2), two transverse fans were used. Pictures of these two fans (wide and narrow transverse fans, and pair of traverse fans) can be seen in Figure 8.

The thicknesses of the PZT and brass shim used in the fans were $0.19 \mathrm{~mm}$ and $0.13 \mathrm{~mm}$, respectively. The cutout in the wide fan was designed to accommodate the power electronics circuitry in its sweep. In the first configuration, the transverse fan was cut narrower to accommodate the sweep of the wide fan during operation. The wide fan has a tip deflection of $0.75 \mathrm{~cm}$ given a $93 \mathrm{~Hz}, 70 \mathrm{~V}$ input, and the narrow transverse fan has a tip deflection of $2 \mathrm{~cm}$ given a $24 \mathrm{~Hz}, 56 \mathrm{~V}$ input. Similarly the transverse fans have tip deflections of approximately $0.75 \mathrm{~cm}$ given a $110 \mathrm{~Hz}, 70 \mathrm{~V}$ input. 
In Figure 9, labels A through D show the locations of the power electronics on which temperatures were monitored during the experiments. Piezoelectric fans were located in the open space $(\mathrm{H})$ between power electronics $\mathrm{A}, \mathrm{B}$, and $\mathrm{C}$ and the $\mathrm{CD}-\mathrm{ROM}$ drive (I). The $\mathrm{CPU}$ fin structure and the rotational fan were located near point $\mathrm{E}$. In each experimental setup, the keyboard of the laptop was replaced with an acrylic cover. This cover had different vents open during the two experiments. In the first setup (wide fan and narrow transverse fan) several small, round vents were opened above the wide fan (four locations marked F in Figure 9). In the second setup, the round vents were closed and a small square vent was opened just above the right transverse fan (location G in Figure 9).

Fourteen thermocouples were used to instrument the setup in these experiments. Four of these were attached to the power electronics, three to the laptop case (one of which was positioned under the CPU), and one to the CPU fin structure. These 8 thermocouples were all fixed in place with an epoxy and their locations remained unchanged throughout the experiment. The remaining six thermocouples were suspended in air, with their locations being different for the different fan placements.

In both experiments the rotational fan inside the laptop turned on automatically when a certain temperature was reached inside the computer. After the laptop reached a steady state with the rotational fan operating, the piezoelectric fans were turned on and a new steady state was achieved.

\section{Setup 1: Wide fan and narrow transverse fan}

The time histories from all the thermocouples used in the experiment are shown in Figure 10. At approximately $1500 \mathrm{~s}$, the rotational fan in the laptop turned on automatically and $1000 \mathrm{~s}$ later, the laptop reached steady-state temperatures. At this time the piezoelectric fans were turned on. An additional $500 \mathrm{~s}$ were required for the laptop to reach a new steady state with both types of fans (rotational and piezoelectric) operating. As expected, the highest temperature in the system was reached on the fin structure above the CPU, with the air temperatures near the power electronics being much lower. It is observed in Figure 10 that although the rotational fan caused a temperature decrease in the CPU fins, its effect on controlling the temperatures of the power electronics was limited.

Fluctuations seen in the fan outlet and vent thermocouples are due to the influence of the outside airflow in the room. After the piezoelectric fans were turned on, all of the air temperature readings showed an increase whereas the fixed thermocouples (those attached to either the laptop case or the power electronics) showed a decrease. This behavior demonstrates that the piezoelectric fans increase the heat transfer coefficient.

The relative cooling effects of the rotational and piezoelectric fans are compared in Figure 11 based on the temperature-history data recorded by the thermocouples. The error bars in this figure represent $\pm 2 \mathrm{~s}$. The 
thermocouple located near the power button shows greater variability than the others (as revealed by the error bars in this figure) since it is exposed to fluctuating ambient conditions. A decrease in temperature of greater than $6^{\circ} \mathrm{C}$ is observed in the middle power electronics for this experiment, solely due to the piezoelectric fans. In addition, a temperature drop of more than $2^{\circ} \mathrm{C}$ was observed in the fin structure mounted on the CPU due to the piezoelectric fans. The effect of the piezoelectric fans on the case temperatures, however, is not significant, partly because their placement on one side of the laptop is somewhat remote from the CPU.

\section{Setup 2: Two transverse fans operating in tandem}

From Figure 12 it is seen that a temperature drop of approximately $6^{\circ} \mathrm{C}$ was observed on all of the power electronics due to the presence of the piezoelectric fans. The air temperature near the vent dropped by more than $8^{\circ} \mathrm{C}$. In this experiment the temperature reduction in the power electronics on the right was significantly greater than in Setup 1, due to the close proximity of the vent. The air temperature near the left end of the cut rib section (labeled $\mathrm{J}$ in Figure 9) increased by approximately $5^{\circ} \mathrm{C}$, reflecting greater mixing due to the piezoelectric fans. A small decrease in temperature was also observed on the CPU fin structure due to the piezoelectric fans. Again, the case temperatures were not significantly altered by the presence of the piezoelectric fans.

\section{Performance Metrics}

In order to design piezoelectric fans for commercial application and operation, it is necessary to characterize their performance. Traditional fan curves for rotary fans are based on the pressure drop across the fans, the power input to the fan, and the airflow and efficiency of the fan [10]. Such fan curves offer information to the designer that can be used to identify a fan that meets the cooling requirements of a system. Piezoelectric fans, however, operate in a manner significantly different from traditional rotational fans. Flow visualization experiments, as well as previous studies [5,6], have shown that flow induced by a vibrating body, such as a piezoelectric fan or a cylinder, can be expressed as a sum of a first-order unsteady term and higher-order steady and unsteady terms. It is the first-order unsteady term that contributes most to the heat transfer enhancement by mixing, whereas the leading order steady term results in the largest net transport of air, thus playing a role in the determination of optimal vent locations. In order to integrate piezoelectric fans into practical cooling applications, they must be better characterized, and their performance metrics established.

A preliminary experiment was performed in the present work to determine whether it was possible to develop fan curves for piezoelectric fans that could be used as a basis for comparison against rotational fans. A 
single fan, measuring $1.27 \mathrm{~cm}$ wide and $8 \mathrm{~cm}$ long, was placed inside several open-ended tubes of increasing length and the velocity was measured at the inlet and outlet of the tubes with a hot wire anemometer. The fan tip deflection was approximately $2 \mathrm{~cm}$ with an input signal of $112 \mathrm{~V}$ and $60 \mathrm{~Hz}$, resulting in a maximum velocity of about $50 \mathrm{~cm} / \mathrm{s}$ at the tip. Pressure drops for these flow rates calculated from conventional correlations based on the measured velocities, tube dimensions, and air properties were on the order of $\mu \mathrm{Pa}$.

It was found that although the outlet velocity generally decreased as tube length increased for given fan operation conditions, the decrease in velocity was not monotonic. The likely reason for this behavior is the fact that the anemometer measurement accounts for both the first-order unsteady and the second-order steady streaming flow. While the steady streaming flow component may exhibit behavior similar to laminar flow in closed channels, the unsteady flow component is more complex. For instance, for the short tubes, alternating vortices at the blade tip cause an unsteady component of flow which is quite significant in relation to the steady flow component at the tube outlet. For longer tubes, these vortices detach from the blade tip and propagate downstream to the tube outlet.

Because piezoelectric fans are primarily recommended for mixing the air near a heat dissipating device, it is reasonable to characterize them based on their (a) efficiency in converting electrical energy into mechanical energy of the fan blade, and (b) heat transfer capabilities, rather than comparing them on the basis of the steady flows as generated by rotational fans. With this approach, for a given thermal management application, a designer would first design the most efficient piezoelectric fan that meets the power and geometry requirements of the system. This can be accomplished by calculating the electromechanical coupling factor (EMCF) for the fan. The EMCF of a given vibration mode of a piezoelectric fan is a measure of the amount of available electrical energy that can be converted to mechanical motion of the fan in that vibration mode. A fan with a larger EMCF will generally increase the performance of a given system by using the input power more efficiently towards transferring heat. Buermann et al. [7] provided a method to design fans with the optimal geometries for maximizing the EMCF. Closed and open circuit frequencies of a piezoelectric fan with symmetrically placed patches were computed both analytically and through a finite element approach, from which the EMCF could be determined.

Figure 13 shows representative results from an optimization problem involving non-dimensionalized piezoelectric fan geometries. The piezomaterial was PZT, the blade shim stock was brass, and the patch was placed flush with the fixed end of the cantilever. In the figure, $\left(\mathrm{L}_{2}-\mathrm{L}_{1}\right)$ is the patch length, $\mathrm{L}_{1}$ is the distance from the edge of the patch to the fixed end of the cantilever (in this case $\mathrm{L}_{1}=0$ ), $\mathrm{L}_{3}$ is the length of the beam, $\mathrm{h}_{\mathrm{p}}$ is the thickness of the patch, and $2 \mathrm{~h}$ is the thickness of the beam. The ratio of the length of the piezoelectric patch to that of the blade 
is referred to as the length ratio, and the ratio of the thickness of a single patch to that of the blade shim stock is referred to as the thickness ratio. From Figure 13 it can be readily seen that EMCF is more sensitive to variations in the length ratio than the thickness ratio. Since the problem is non-dimensionalized, a piezoelectric fan could nominally be designed from this approach for applications of any size by scaling the geometries. However, if it is desired that the fan be noiseless, care must be taken to ensure that the geometry of the fan leads to a resonant frequency outside the audible range. It may also be noted that the optimal geometries for maximal EMCF depend on the combination of materials used for the piezoelectric patch and the fan blade. Figure 14 shows EMCF results if the placement of the patch, rather than the thickness ratio, is allowed to vary. It is easily observed that placement of the patch flush to the fixed end of the cantilever results in the fan with maximal EMCF.

Once optimization is completed and the fan constructed, the designer must determine the thermal performance or convection heat transfer coefficient. These parameters are, however, affected by (a) the size of the enclosure, (b) the proximity of the fan to the heat dissipating device, (c) the location of vents and outlets, and (d) the dimensions of the fans of a specific system. The system must be built and the fan implemented in order to find the optimal position for maximizing heat transfer. As was demonstrated in the cell phone enclosure experiment, positioning of the piezoelectric fan relative to the heat dissipating device is an important consideration.

\section{Conclusions and Recommendations}

It has been shown that piezoelectric fans are a viable technology for meeting a variety of cooling needs in electronic devices. They are small, noiseless, low-power devices, and can easily be fabricated to suit specific applications. They are not intended to replace rotary fans in larger devices, such as laptops and personal computers, but can provide supplemental cooling in hot spots and other stagnant areas where a rotary fan is ineffective. In smaller devices, where rotary fans are not practical and electronics are pushed to the limits of their heat dissipation capacities, piezoelectric fans offer a realistic cooling solution while meeting the noise and power requirements of portable devices.

Additional work needs to be accomplished in the modeling and optimization of these fans, particularly for understanding the flow induced by the fans and the interaction of the flow with the surroundings. This will not only enable the effects of the surrounding electronics to be coupled to the fan placement, and optimal locations for the fans to be identified, but will also lead to better and more efficient fan designs. 


\section{Acknowledgements}

The authors acknowledge the financial support from members of the Cooling Technologies Research Center (http://widget.ecn.purdue.edu/ CTRC), a National Science Foundation Industry/University Cooperative Research Center at Purdue University.

\section{References}

[1] Toda, M., Voltage-induced Large Amplitude Bending Device-PVF 2 Bimorph-Its Properties and Applications, Ferroelectrics, Vol. 32, pp 127-133, 1981.

[2] Toda, M., Theory of Air Flow Generation by a Resonant Type $\mathrm{PVF}_{2}$ Bimorph Cantilever Vibrator, Ferroelectrics, Vol. 22, pp 911-918, 1979.

[3] Yoo, J. H., Hong, J. I., and Cao, W., Piezoelectric Ceramic Bimorph Coupled to Thin Metal Plate as Cooling Fan for Electronic Devices, Sensors and Actuators A, Vol. 79, pp 8-12, 2000.

[4] Schmidt, R. R., Local and Average Transfer Coefficients on a Vertical Surface Due to Convection from a Piezoelectric Fan, InterSociety Conference on Thermal Phenomena, pp 41- 49, 1994.

[5] Loh, B. G., Hyun, S., Ro, P. I., and Kleinstreuer, C., Acoustic Streaming Induced by Ultrasonic Flexural Vibrations and Associated Enhancement of Convective Heat Transfer, Journal of Acoustical Society of America, Vol. 111, pp 875$883,2002$.

[6] Nyborg, W. L., Acoustic Streaming near a Boundary, Journal of Acoustical Society of America, Vol. 30, pp 329-339, 1958.

[7] Buermann, P., Raman A., and Garimella, S. V., Dynamics and Topology Optimization of Piezoelectric Fans, IEEE Transactions on Components and Packaging Technologies (in press); also in Thermal Challenges in Next Generation Electronic Systems: THERMES 2002, pp. 113-121, Millpress, Netherlands, January 2002.

[8] Ihara, A., and Watanabe, H., On the Flow around Flexible Plates, Oscillating With Large Amplitude, Journal of Fluids and Structures, Vol. 8, pp 601-619, 1994.

[9] Garimella, S. V, and Eibeck, P. A., Heat Transfer Characteristics of an Array of Protruding Elements in Single Phase Forced Convection, International Journal of Heat and Mass Transfer, Vol. 33, No. 12, pp. 2659-2669, 1990.

[10] American National Standards Institute, Laboratory Methods of Testing Fans for Aerodynamic Performance Rating, ANSI/AMCA Standard 210-99, 1999. 


\section{List of Figures}

Figure $\quad$ Page

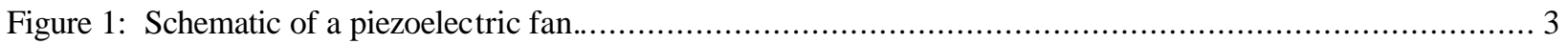

Figure 2: Prototype piezoelectric fans. The acrylic cover and vanes allow control of flow directionality.............. 4

Figure 3: Schematic of the experimental setup to visualize near-boundary flow. View direction for the visualization

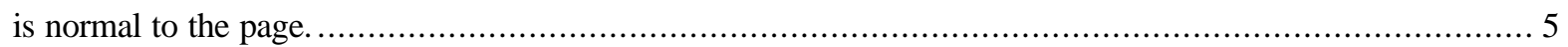

Figure 4: Transient flow field from near-boundary flow visualization experiments, at times of (a) 1/30, (b) 2/30, (c) 3/30, and (d) $5 / 30$, (e) $8 / 30$, (f) $10 / 30$, (g) $13 / 30$ and (h) $15 / 30 \mathrm{~s}$. The fan blade position is shown in (a)........ 6

Figure 5: Estimated streamlines for developed flow from the near-boundary flow visualizations....................... 8

Figure 6: Experimental setup for the cell phone cooling experiment. Three different positions were tested in the vertical fan orientation: Half-coverage over heat sink (position 1); full coverage over heat sink (position 2); no coverage over heat sink (position 3). One position was tested in the horizontal fan orientation (position 4).... 9

Figure 7: Enhancement in heat transfer coefficient for the four different piezoelectric fan positions in the cell phone enclosure. At $t=0 \mathrm{~s}$, the piezoelectric fan inside the enclosure is turned on. The system reached steady state

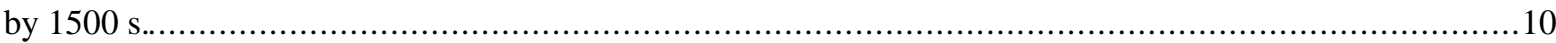

Figure 8: Piezoelectric fans used in the laptop cooling experiments. On the left are the wide fan and narrow transverse fan (used in combination), and on the right are the transverse fans....................................11

Figure 9: Photograph of the laptop used in cooling experiment. A-D show the location of the power electronics, E is the CPU fin structure, F and G show the placement of vents in setups 1 and 2, respectively, and I shows the location of the CD-ROM drive. The piezoelectric fans were placed in the open space $(\mathrm{H})$ between the power electronics and the CD-ROM drive.

Figure 10: Temperature versus time graph for thermocouples used in Setup 1 (wide fan and narrow transverse fan with open vent above wide fan). At $t=1500 \mathrm{~s}$, the rotary fan inside the laptop turns on automatically. At $\mathrm{t}=$ $2500 \mathrm{~s}$, the piezoelectric fans are turned on manually.

Figure 11: Change in temperature in Setup 1 due to the rotational fan and the piezoelectric fans. The white bars represent contributions to temperature change due to the rotational fan, and the contributions from the piezoelectric fans are shown as cross-hatched bars. Error bars represent $\pm 2 \sigma$. 
Figure 12: Change in temperature in Setup 2 due to the rotational fan and the piezoelectric fans. The white bars represent contributions to temperature change due to the rotational fan, and the contributions from the piezoelectric fans are shown as cross-hatched bars. Error bars represent $\pm 2 \sigma$.

Figure 13: Optimization of EMCF based on length and thickness ratios. $\mathrm{L}_{2}-\mathrm{L}_{1}$ is the length of the piezoelectric patch, $L_{3}$ is the length of the fan blade, $h_{p}$ is the thickness of the piezoelectric patch, and $2 h$ is the thickness of the fan blade. In this case, the patch is located flush to the fixed edge of the cantilever. Adapted from [7].....16 Figure 14: Optimization of EMCF based on patch placement and thickness ratio. $\mathrm{L}_{2}-\mathrm{L}_{1}$ is the length of the piezoelectric patch, $\mathrm{L}_{1}$ is the distance from the patch to the fixed end of the cantilever, $\mathrm{L}_{3}$ is the length of the fan blade, $h_{p}$ is the thickness of the piezoelectric patch, and $2 \mathrm{~h}$ is the thickness of the fan blade. Adapted from

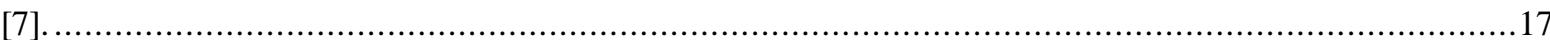




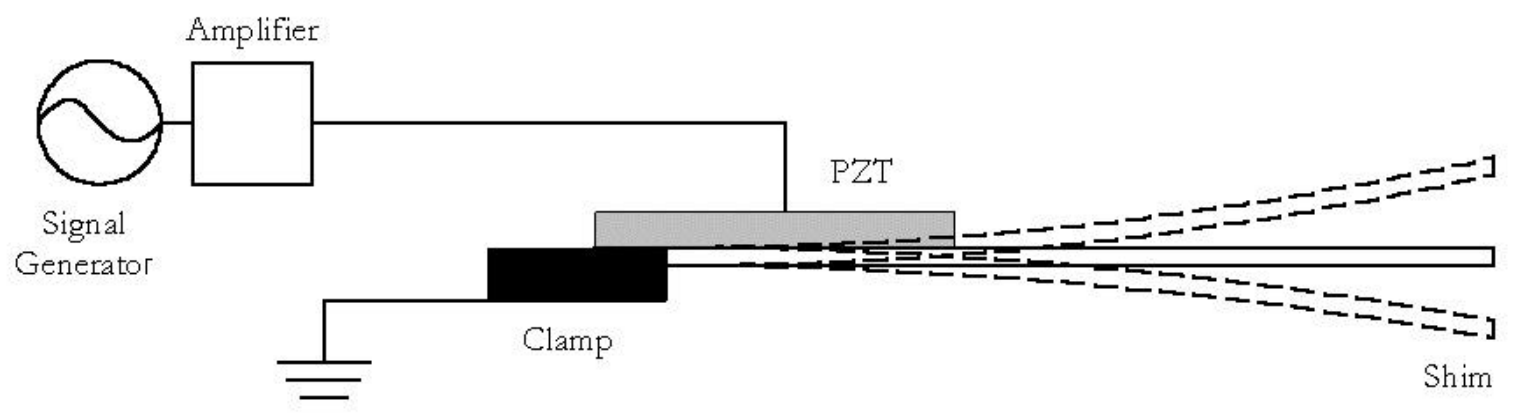

Figure 1: Schematic of a piezoelectric fan. 


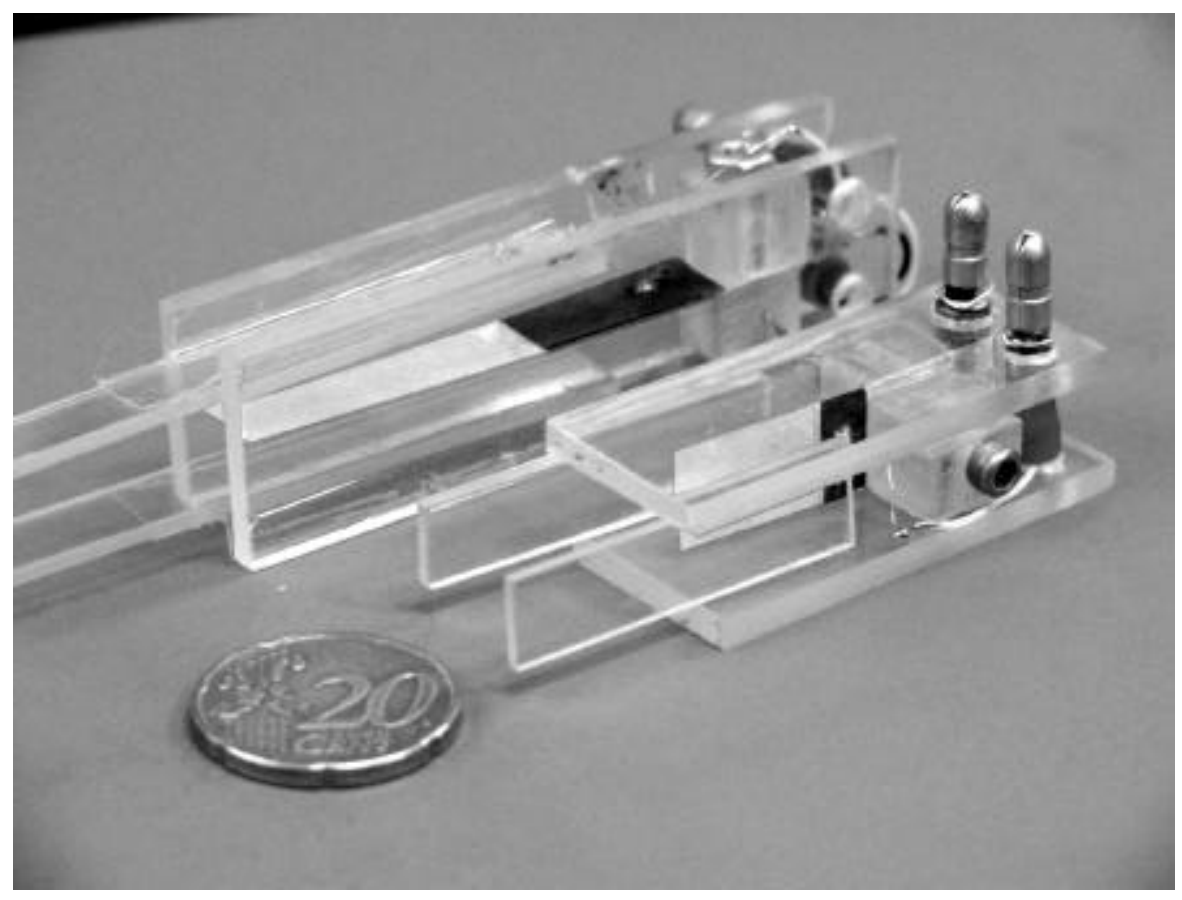

Figure 2: Prototype piezoelectric fans. The acrylic cover and vanes allow control of flow directionality. 


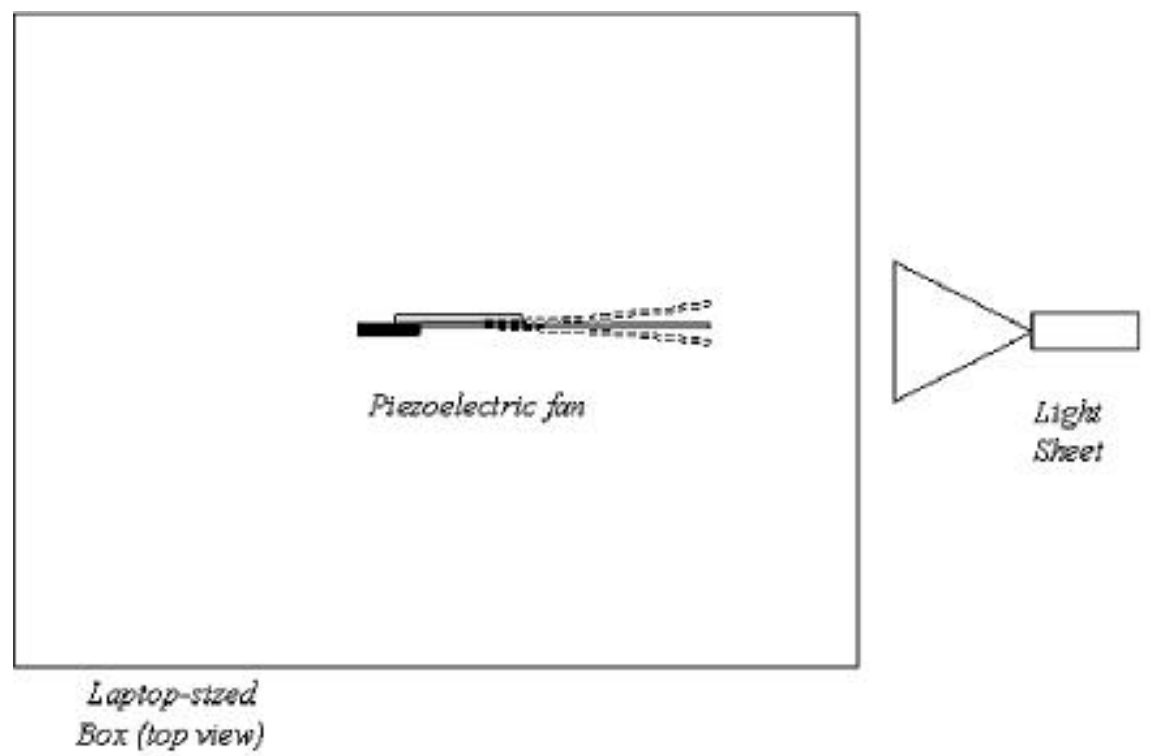

Figure 3: Schematic of the experimental setup to visualize near-boundary flow. View direction for the visualization is normal to the page. 


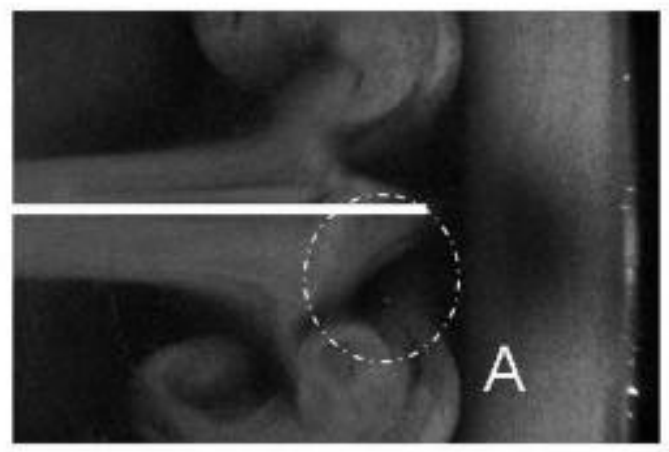

(a) $t=1 / 30 \mathrm{sec}$

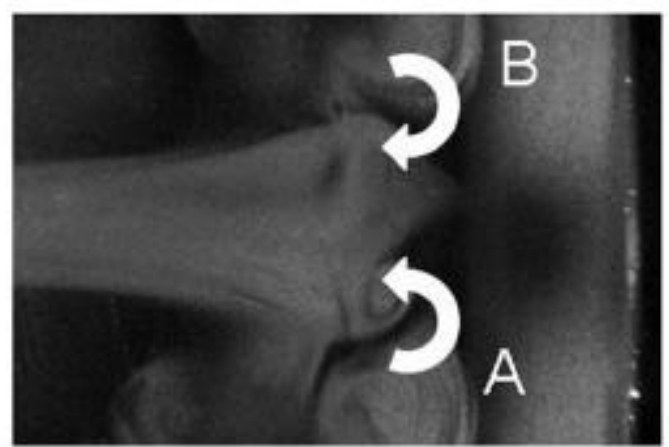

(c) $t=3 / 30 \mathrm{sec}$

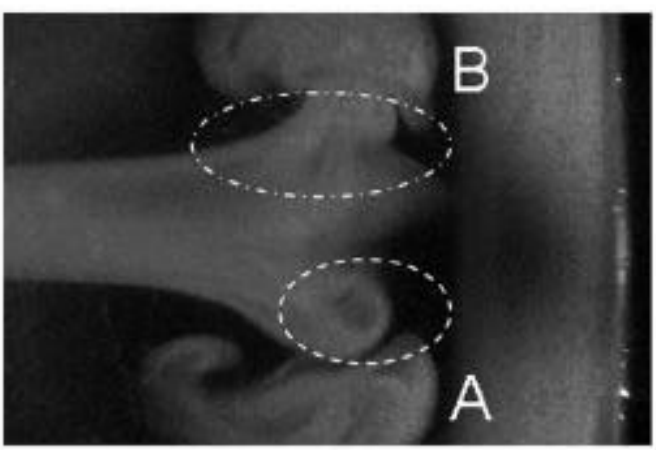

(b) $t=2 / 30 \mathrm{sec}$

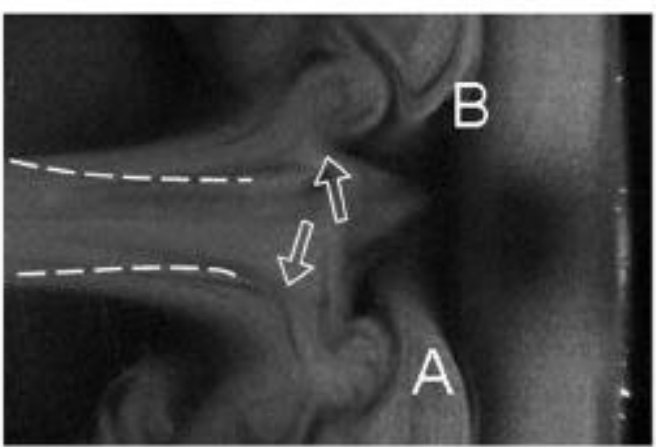

(d) $t=5 / 30 \mathrm{sec}$

Figure 4: Transient flow field from near-boundary flow visualization experiments, at times of (a) $1 / 30$, (b) $2 / 30$, (c) $3 / 30$, and (d) $5 / 30$, (e) $8 / 30$, (f) $10 / 30$, (g) $13 / 30$ and (h) $15 / 30 \mathrm{~s}$. The fan blade position is shown in (a). 


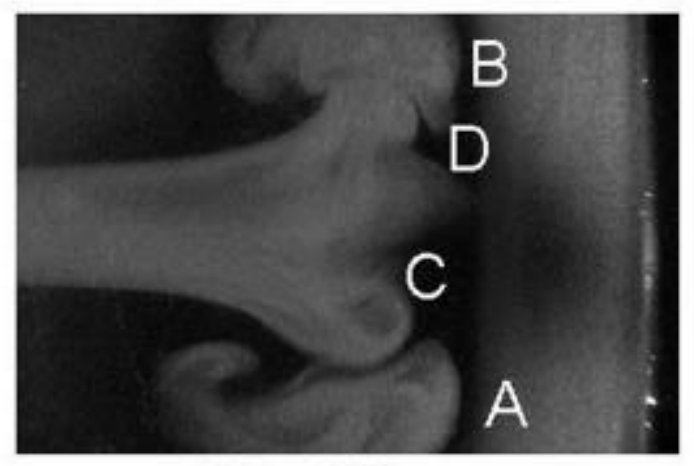

(e) $t=8 / 30 \mathrm{sec}$

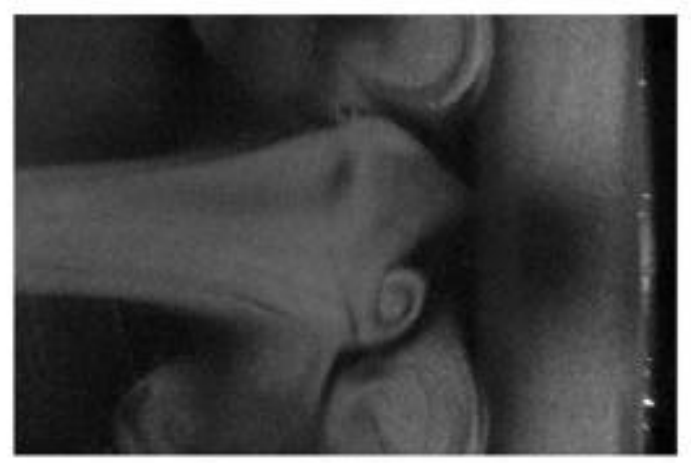

(g) $t=13 / 30 \mathrm{sec}$

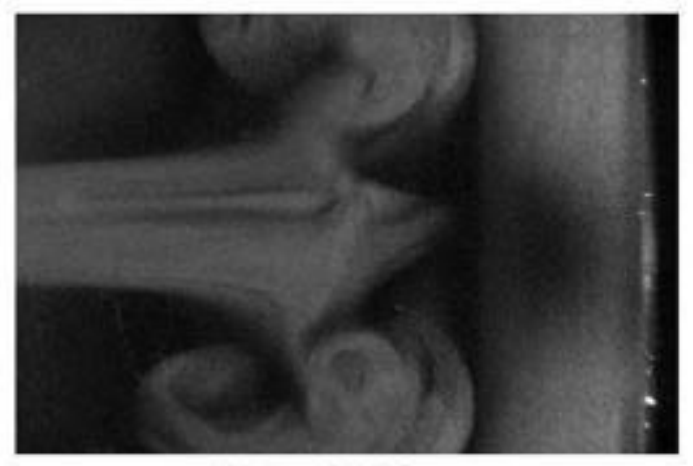

(f) $t=10 / 30 \mathrm{sec}$

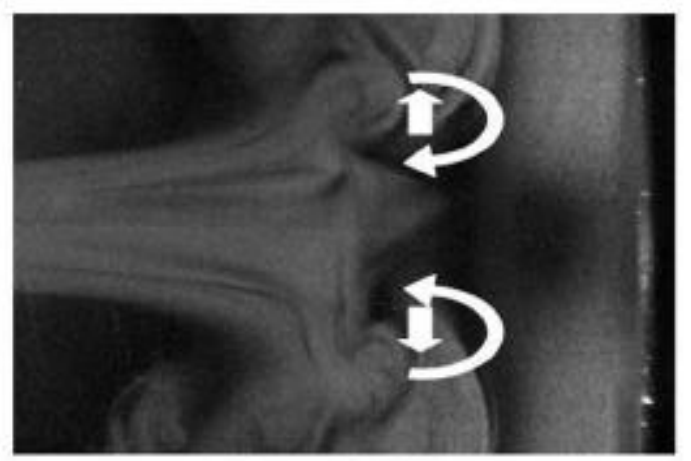

(h) $t=15 / 30 \mathrm{sec}$

Figure 4: Transient flow field from near-boundary flow visualization experiments, at times of (a) $1 / 30$, (b) $2 / 30$, (c) $3 / 30$, and (d) 5/30, (e) $8 / 30$, (f) 10/30, (g) $13 / 30$ and (h) $15 / 30 \mathrm{~s}$. The fan blade position is shown in (a). 


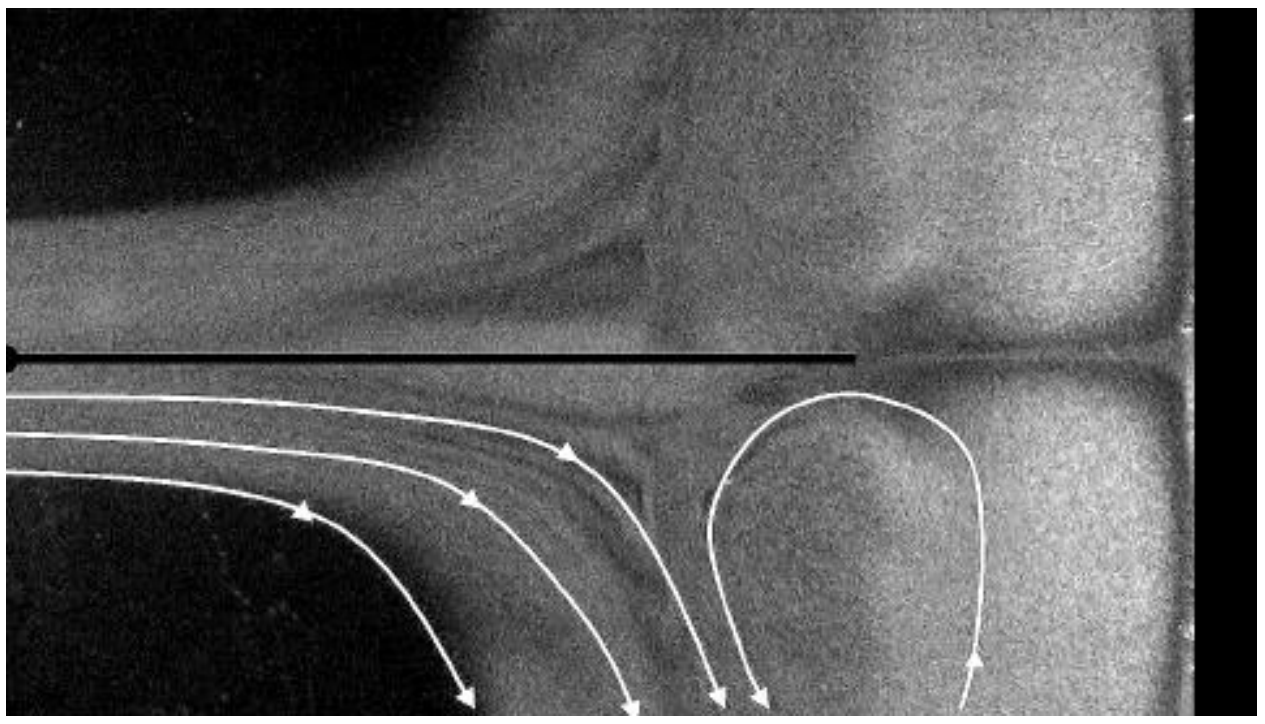

Figure 5: Estimated streamlines for developed flow from the near-boundary flow visualizations. 


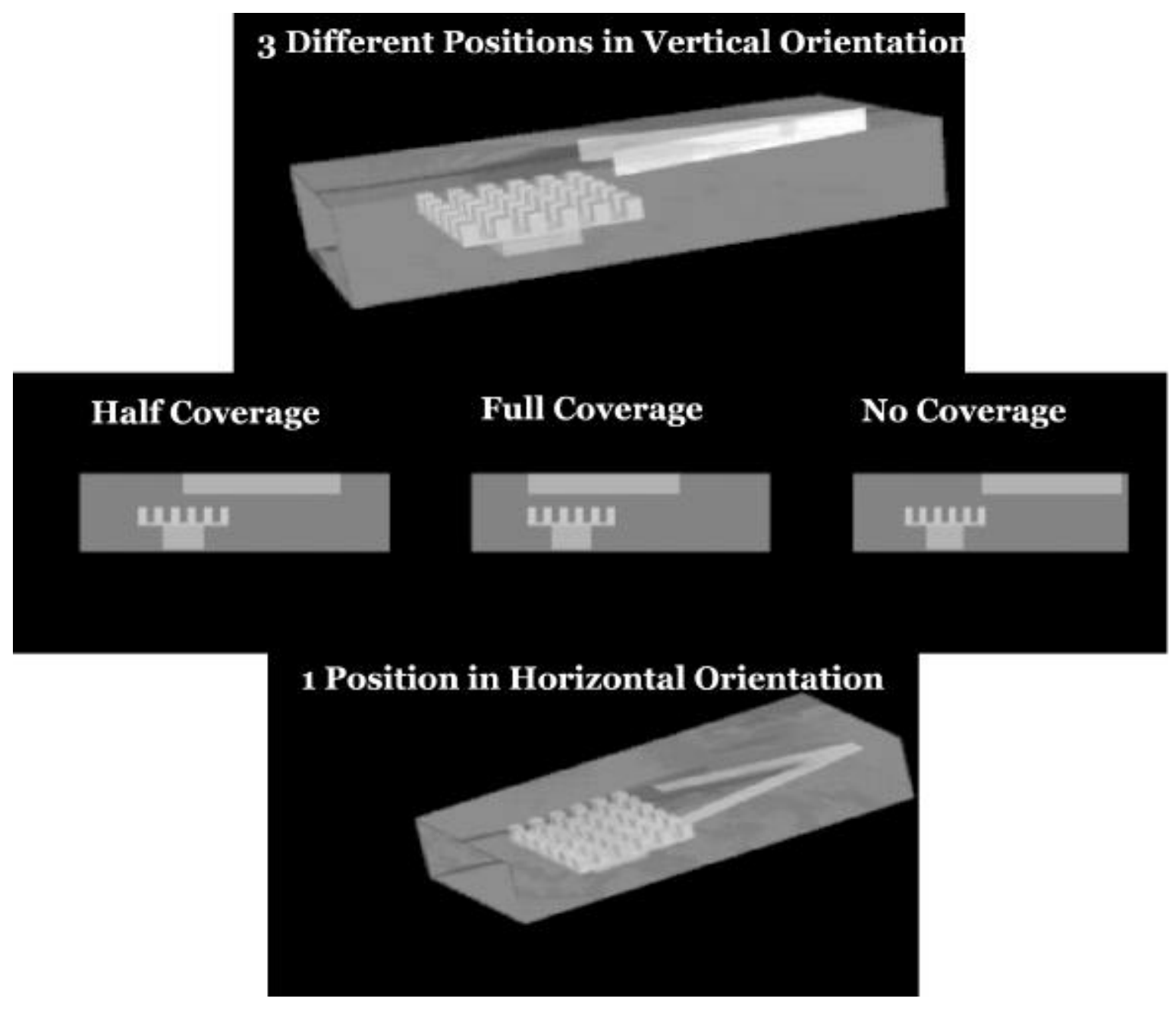

Figure 6: Experimental setup for the cell phone cooling experiment. Three different positions were tested in the vertical fan orientation: Half-coverage over heat sink (position 1); full coverage over heat sink (position 2); no coverage over heat sink (position 3). One position was tested in the horizontal fan orientation (position 4). 


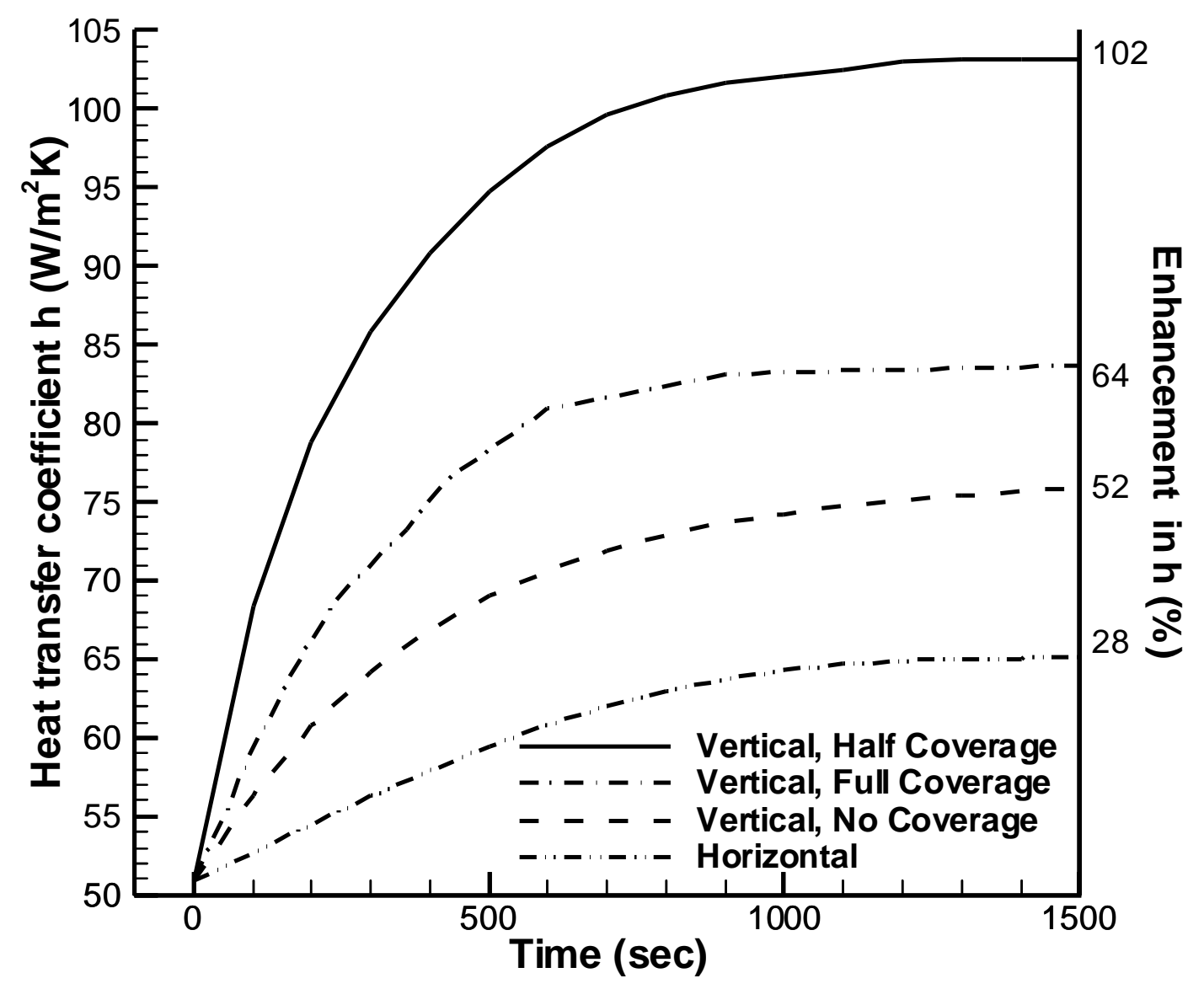

Figure 7: Enhancement in heat transfer coefficient for the four different piezoelectric fan positions in the cell phone enclosure. At $\mathrm{t}=0 \mathrm{~s}$, the piezoelectric fan inside the enclosure is turned on. The system reached steady state by $1500 \mathrm{~s}$. 

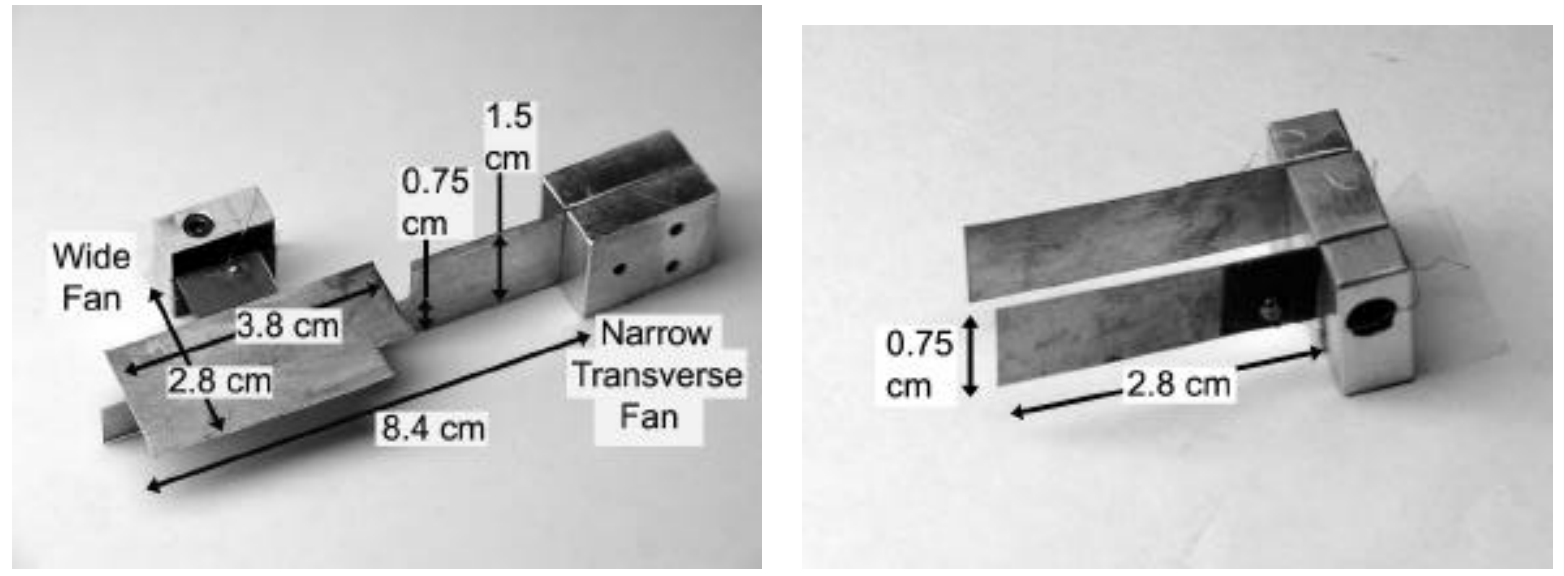

Figure 8: Piezoelectric fans used in the laptop cooling experiments. On the left are the wide fan and narrow transverse fan (used in combination), and on the right are the transverse fans. 


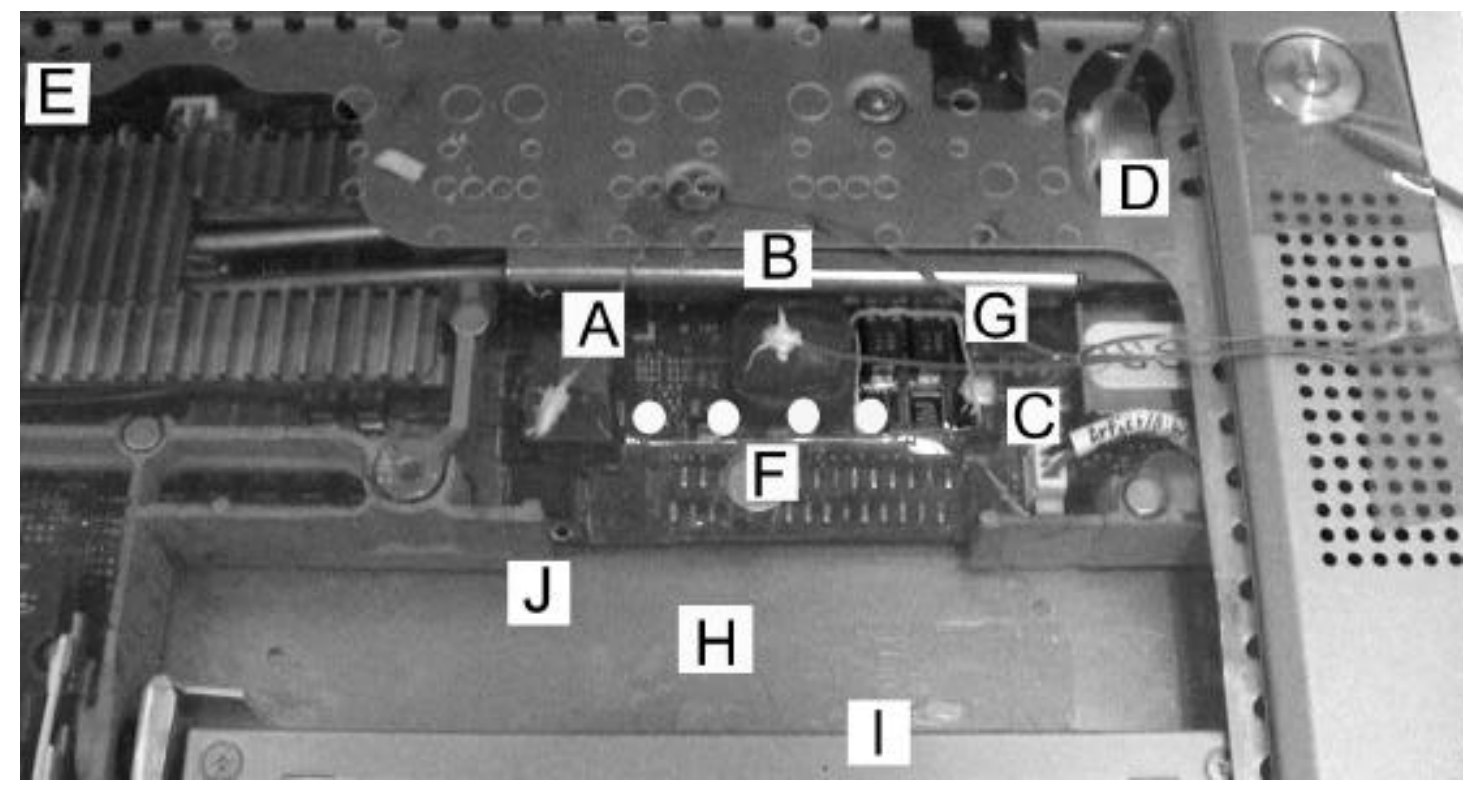

Figure 9: Photograph of the laptop used in cooling experiment. A-D show the location of the power electronics, $\mathrm{E}$ is the $\mathrm{CPU}$ fin structure, $\mathrm{F}$ and $\mathrm{G}$ show the placement of vents in setups 1 and 2, respectively, and I shows the location of the CD-ROM drive. The piezoelectric fans were placed in the open space $(\mathrm{H})$ between the power electronics and the CD-ROM drive. 


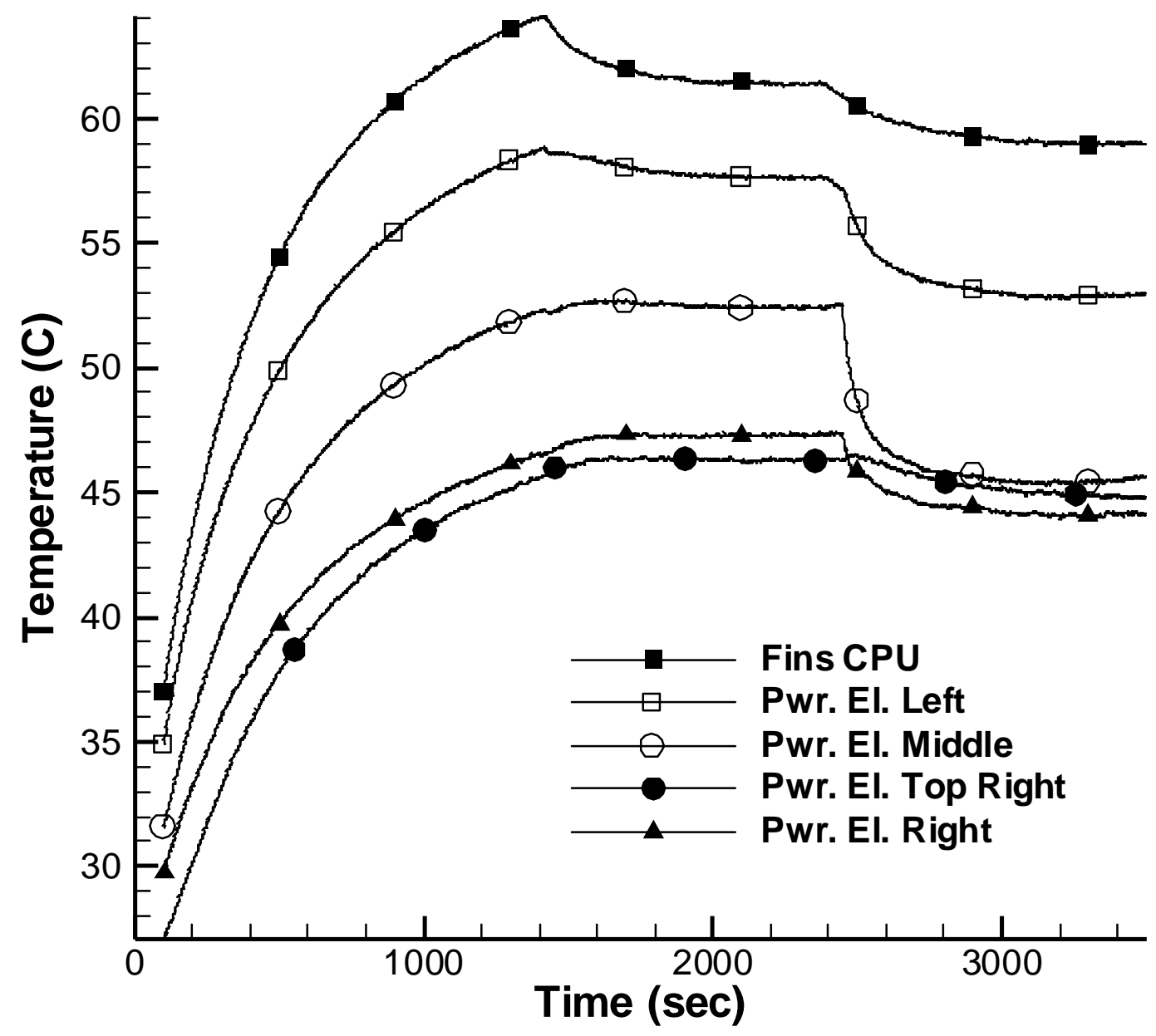

Figure 10: Temperature versus time graph for thermocouples used in Setup 1 (wide fan and narrow transverse fan with open vent above wide fan). At $t=1500 \mathrm{~s}$, the rotary fan inside the laptop turns on automatically. At $\mathrm{t}=2500 \mathrm{~s}$, the piezoelectric fans are turned on manually. 


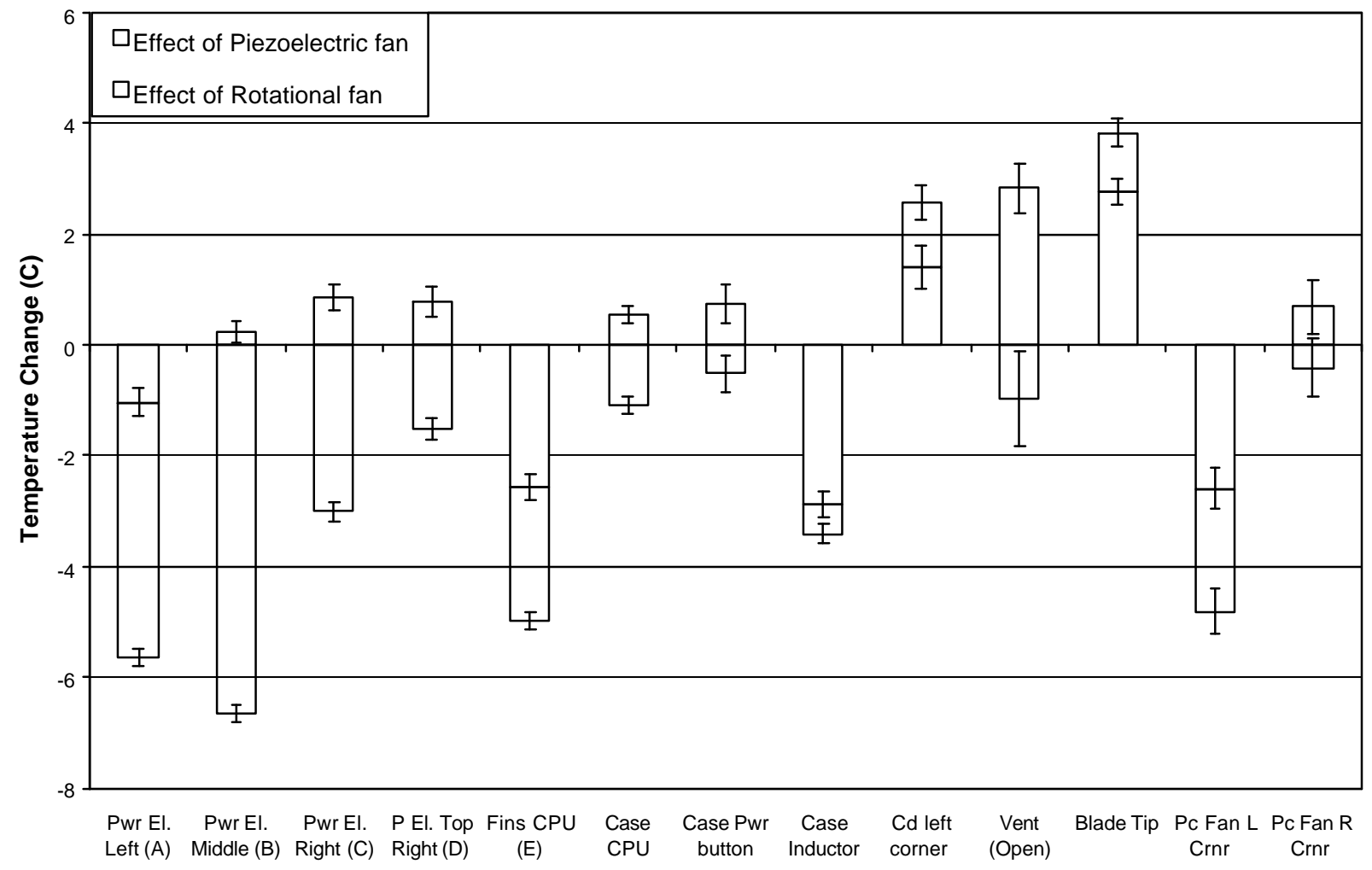

Figure 11: Change in temperature in Setup 1 due to the rotational fan and the piezoelectric fans. The white bars represent contributions to temperature change due to the rotational fan, and the contributions from the piezoelectric fans are shown as cross-hatched bars.

Error bars represent $\pm 2 \sigma$. 


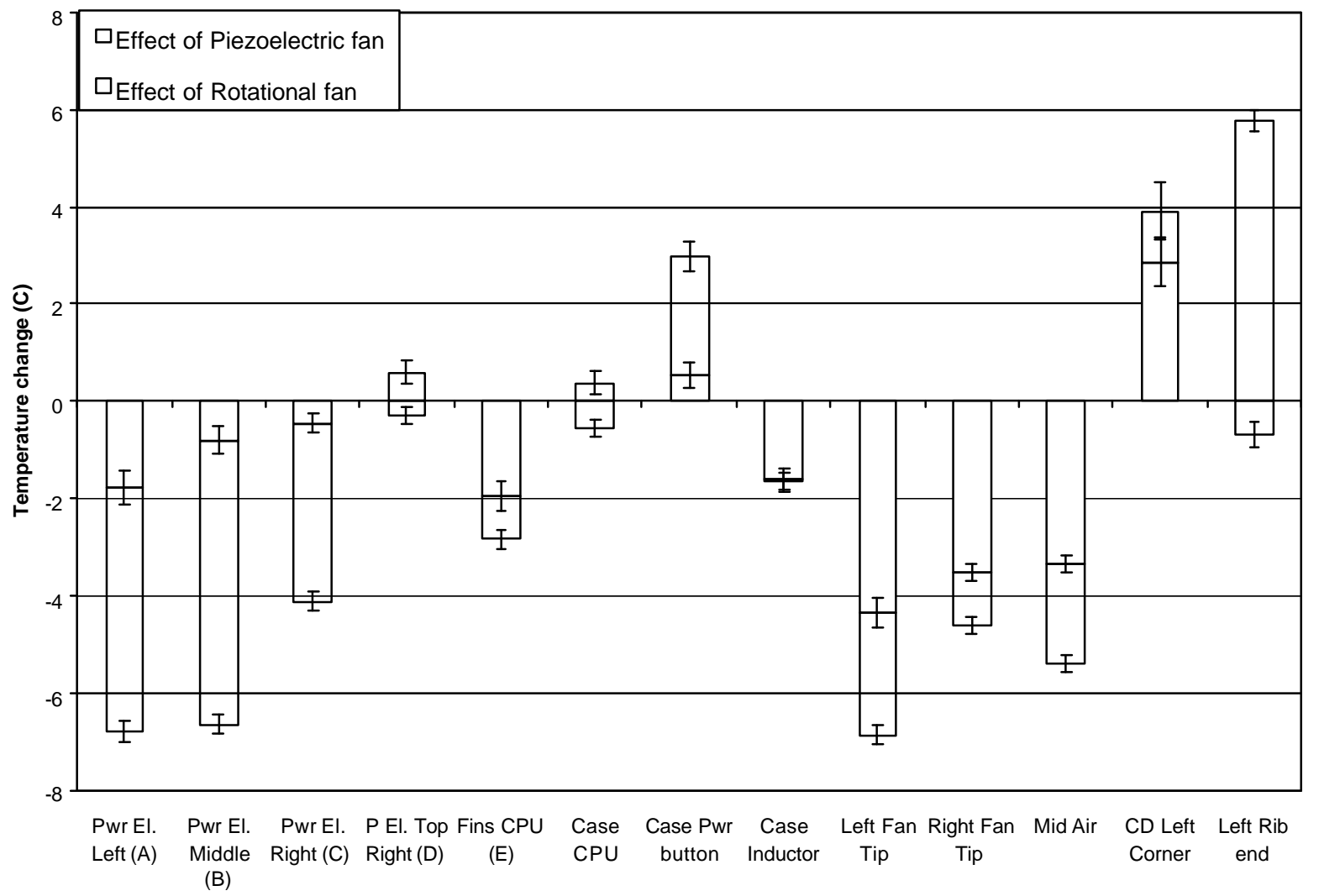

Figure 12: Change in temperature in Setup 2 due to the rotational fan and the piezoelectric fans. The white bars represent contributions to temperature change due to the rotational fan, and the contributions from the piezoelectric fans are shown as cross-hatched bars.

Error bars represent $\pm 2 \sigma$. 


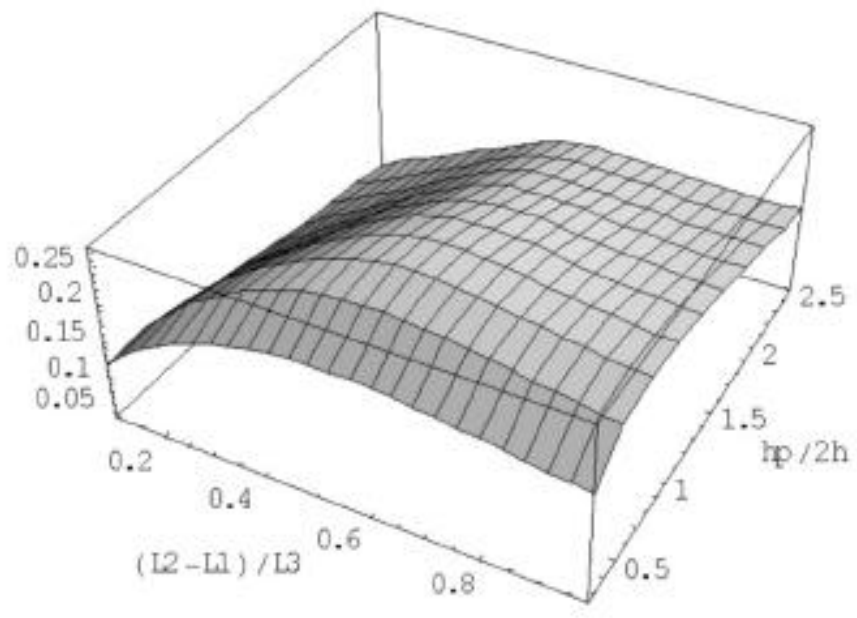

Figure 13: Optimization of EMCF based on length and thickness ratios. $\mathrm{L}_{2}-\mathrm{L}_{1}$ is the length of the piezoelectric patch, $L_{3}$ is the length of the fan blade, $h_{p}$ is the thickness of the piezoelectric patch, and $2 \mathrm{~h}$ is the thickness of the fan blade. In this case, the patch is located flush to the fixed edge of the cantilever. Adapted from [7]. 


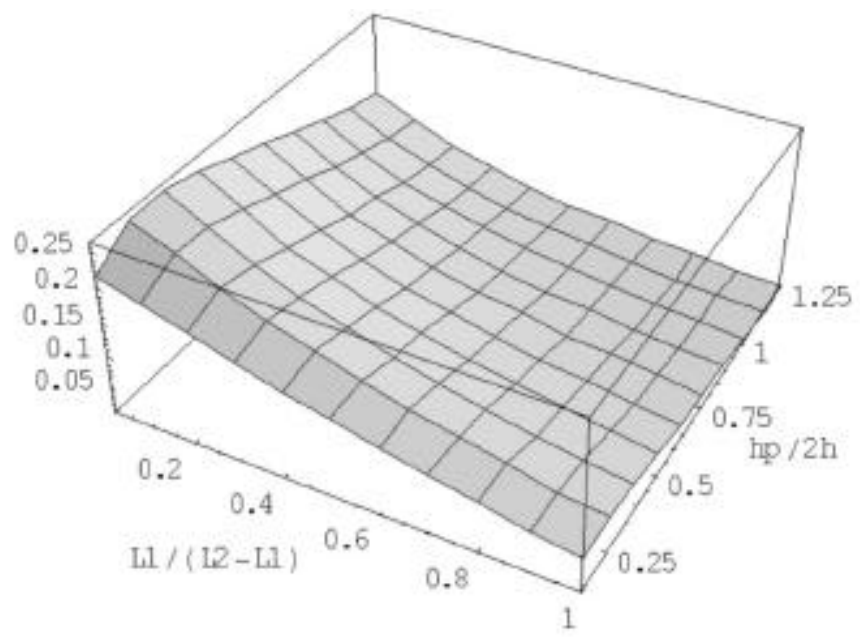

Figure 14: Optimization of EMCF based on patch placement and thickness ratio. $\mathrm{L}_{2}-\mathrm{L}_{1}$ is the length of the piezoelectric patch, $\mathrm{L}_{1}$ is the distance from the patch to the fixed end of the cantilever, $h_{p}$ is the thickness of the piezoelectric patch, and $2 \mathrm{~h}$ is the thickness of the fan blade. Adapted from [7]. 